\title{
Atypical Goblet Cell Hyperplasia in Congenital Cystic Adenomatoid Malformation as a Possible Preneoplasia for Pulmonary Adenocarcinoma in Childhood: A Genetic Analysis
}

\author{
ELVIRA STACHER, MD, REINHARD ULLMANN, PHD, IRIS HALBWEDL, \\ MARGIT GOGG-KAMMERER, LILIANE BOCCON-GIBOD, MD, \\ ANDREW G. NICHOLSON, MD, MARY N. SHEPPARD, MD, \\ LINA CARVALHO, MD, MARIA TERESA FRANCA, MD, \\ FERGUS MACSWEENEY, MD, ALICIA MORRESI-HAUF, MD, AND \\ HELMUT H. POPPER, MD
}

\begin{abstract}
Congenital cystic adenomatoid malformation (CCAM) of the lung is a congenital lesion that is sometimes complicated by bronchioloalveolar adenocarcinoma (BAC). In some cases foci of atypical goblet cell hyperplasia (AGCH) can be found within the cysts. It has been proposed that CCAM and AGCH predispose to the development of BAC. The present study used comparative genomic hybridization (CGH) to screen 22 cases of CCAM (epithelium, surrounding normal lung tissue, and both preneoplastic and neoplastic lesions) for chromosomal imbalances. Of these 22 cases, 10 were CCAM type 1,10 were type 2 , and 2 were type 3 . Of the 10 cases of CCAM type 1,2 were associated with AGCH, 1 was associated with atypical adenomatous hyperplasia (AAH) and associated tubular adenocarcinoma (AC), and 2 were associated with BAC (1 mucinous and 1 predominantly nonmucinous). The present study also involved immunohistochemistry for interleukin (IL)-13, IL-4 receptor- $\alpha$ (IL-4r $\alpha$ ), cytokines involved in the differentiation of goblet cells, and mucin 2 protein (Muc2). Chromosomal aberrations were not detected in the epithelium or the surrounding normal lung tissue, whereas varying
\end{abstract}

Congenital cystic adenomatoid malformations (CCAMs) are rare cystic lesions arising in the lung that were first grouped into 3 types based on their gross appearance: cystic, intermediate, and solid. ${ }^{1}$ Stocker later recommended that they be renamed types 1,2 ,

From the Institute of Pathology, University of Graz, Graz, Austria; Childrens Hospital Armand Trousseau, Paris, France; Department of Pathology, Royal Brompton Hospital, London, UK; Institute of Anatomic Pathology, University of Coimbra, Coimbra, Portugal; Institute of Anatomic Pathology, Hospital of Santa Martha, Lisboa, Portugal; and Institute of Pathology, Special Clinics Asklepios, Gauting, Germany. Accepted for publication January 7, 2004.

Supported by the Hans und Blanca Moser Foundation (E.S.) and the Austrian Research Fund, project P14759 (R.U. and I.H.).

Address correspondence and reprint requests to Helmut $\mathrm{H}$. Popper, MD, Laboratories for Molecular Cytogenetics, Environmental and Respiratory Pathology, Institute of Pathology, Auenbruggerplatz 25, Graz A-8036, Austria.

0046-8177/\$—see front matter

(C) 2004 Elsevier Inc. All rights reserved.

doi:10.1016/j.humpath.2004.01.008 aberrations were found in the neoplastic lesions. The most frequent genomic imbalances observed in both AGCH and the carcinomas were gains in chromosomes 2 and 4 . Interestingly, a predominance of gains was also reported in AC of nonsmokers. Chromosomal aberrations in AGCHs arising in CCAMs support their preneoplastic status. Nuclear expression of IL-13, IL-4r $\alpha$, and Muc2 was detected in AGCH, whereas a cytoplasmic and nuclear reaction was seen in normal epithelium. This likely reflects an association with goblet cell differentiation, but it also drives proliferation in AGCH. Hum Pathol 35:565-570. ㅇ 2004 Elsevier Inc. All rights reserved.

Key words: congenital cystic adenomatoid malformation, atypical goblet cell hyperplasia, preneoplasia, adenocarcinoma, comparative genomic hybridization.

Abbreviations: AAH, atypical adenomatous hyperplasia; AC, adenocarcinoma; AGCH, atypical goblet cell hyperplasia; BAC, bronchioloalveolar adenocarcinoma; CCAM, congenital cystic adenomatoid malformation; CGH, comparative genomic hybridization; DOP-PCR, degenerate oligonucleotide primer polymerase chain reaction.

and $3,{ }^{2}$ and later added types 0 and $4,{ }^{3}$ envisioning that the enlarged spectrum represented malformations of 5 successive groups of airways.

The pathogenesis of CCAMs is uncertain, but it has been suggested that they represent maturation defects at various points during organogenesis and differentiation. They are diagnosed mainly in stillborn fetuses or newborn infants, but sometimes are not detected until adulthood. ${ }^{4}$ Prenatal ultrasound scanning has also allowed their detection in utero. ${ }^{5}$ Rare cases of CCAM show malignant transformation, usually bronchioloalveolar adenocarcinoma (BAC) within a type 1 lesion. ${ }^{6}$

In addition to BAC, type 1 CCAMs demonstrate focal atypical goblet cell hyperplasia (AGCH) in approximately $33 \%$ of cases, ${ }^{7}$ although the incidence of carcinomatous transformation is less than $1 \%$. However, the only evidence that these lesions represent true malignancies is the rare incidence of metastatic spread. $^{8}$ Furthermore, the relationship between $\mathrm{AGCH}$ and BAC in terms of carcinogenesis is uncertain. 
TABLE 1. Clinical Data for the 5 Patients With Preneoplastic Lesions or Adenocarcinoma

\begin{tabular}{lllll}
\hline & Age & Sex & \multicolumn{1}{c}{ Clinical presentation } & \multicolumn{1}{c}{ Follow-up } \\
\hline Patient 1 & 3 years & M & Cystic lung lesion & Followed-up for 3 years; fd \\
Patient 2 & Newborn & F & Cystic lung lesion & fd \\
Patient 3 & 13 years & M & Cystic lung lesion, suspicious tumor & Follow-up for 11 years; fd \\
Patient 4 & 29 years & M & Tumor, cystic lung lesion & Follow-up for 2 years; alive, pd \\
Patient 5 & 3 years & F & Cystic lung lesion, nodular lesion & Follow-up for 16 years; fd \\
\hline
\end{tabular}

Abbreviations: fd, free of disease; pd, progressive disease.

Consequently, the primary aim of the present study was to investigate chromosomal aberrations in different types of CCAM, comparing changes in areas of AGCH and BAC with the surrounding cysts and lung parenchyma. In addition, because it is known that interleukin (IL)-13 is up-regulated in inflamed tissue and induces differentiation into goblet cells in animal models, ${ }^{9}$ with up-regulation of mucin 2 gene (Muc2) via a pathway that involves the IL-4 receptor $\alpha(\mathrm{IL}-4 \mathrm{R} \alpha),{ }^{10}$ this study also analyzed the expression of IL-13, IL-4R $\alpha$, and Muc2 within areas of goblet cell proliferation.

\section{MATERIAL AND METHODS}

\section{Study Samples}

Participants in the European Project on Rare Pulmonary Diseases (funded by the Health \& Consumer Protection Directorate General of the European Commission; project 2001/RD/10010) collected 43 cases of CCAM as formalinfixed, paraffin-embedded tissue blocks. Fourteen of these specimens were CCAM type 1,25 were CCAM type 2 , and 4 were CCAM type 3. Two pathologists independently reviewed the typing of CCAM. No cases of types 0 and 4 were identified. Five cases of CCAM type 1 were associated with an AGCH, 1 case of CCAM type 1 was combined with a multifocal atypical adenomatous hyperplasia (AAH), and 5 cases of CCAM type 1 were associated with AC. Patient age ranged from 27 gestational weeks to 41 years.

Sixteen cases demonstrating either significant inflammation or massive hemorrhage were rejected, including cases with degraded DNA. In 2 cases with ACs and 3 cases with AGCH present in cysts, there was insufficient material for analysis by comparative genomic hybridization $(\mathrm{CGH}) .{ }^{11}$ This left 22 cases for analysis, including 3 cases associated with foci of AC, 1 case containing foci of AAH, and 2 cases with cysts containing foci of AGCH (Table 1).

The tumor samples were examined and classified by an experienced pathologist. Sections for laser microdissection (Palm, Bernried, Germany) were stained with diluted Harris hematoxylin solution (Merck, Darmstadt, Germany).
In each case, epithelium lining the cysts and surrounding normal lung tissue, as well as areas of AGCH and BAC when present, were analyzed separately by CGH. AGCH was diagnosed if mucous cell proliferations were found embedded in the epithelium of the cysts or as small foci in adjacent alveoli. BAC was diagnosed when atypical cells formed single rows of cells on alveolar walls completely replacing the normal epithelium.

\section{Comparative Genomic Hybridization}

Genomic DNA from the tumors was extracted using standard proteinase $\mathrm{K}$ digestion. DNA was amplified by degenerate oligonucleotide primer polymerase chain reaction (DOPPCR) as described previously. ${ }^{12}$ In each round of DOP-PCR, a normal control was coamplified and also analyzed using CGH. All hybridizations were performed in a sex-mismatch manner as an internal control. The Quips 3.1 software (Vysis, Downers Grove, IL) was used for CGH analysis. The wellknown critical regions for CGH-1p36, 17p, 19p, 22, and the telomeric and centromeric regions-were not analyzed. Only cases where the sex mismatch was clearly identified by CGH were included. For each tumor sample, an average of 10 metaphase spreads was examined. Thresholds were set to 0.8 and 1.2 .

\section{Immunohistochemistry}

Anti-IL-13, anti-IL-4R $\alpha$, and anti-Muc2 (Santa Cruz Biotechnology, Santa Cruz, CA) were used as the primary antibodies as recommended. The incubation, washing steps, and detection procedures were performed in routine fashion. Microwave pretreatment was applied, with diaminobenzidine (ChemMed; Dako, Glostrup, Denmark) used as a chromogen, yielding a brown reaction product. Nuclei were counterstained slightly with hematoxylin.

\section{RESULTS}

\section{Comparative Genomic Hybridization}

Twenty-two cases of CCAM (10 type 1, 10 type 2, and 2 type 3) were analyzed by CGH. Two cases of

FIGURE 1. CCAM type 1 with $A G C H$ inside the cyst (patient 1). (Hematoxylin and eosin; original magnification $\times 100$.)

FIGURE 2. AGCH within normal lung, adjacent to CCAM cysts (patient 2). (Hematoxylin and eosin; original magnification $\times 50$.)

FIGURE 3. AAH in normal lung adjacent to a CCAM cyst (patient 3). (A) Overview showing 4 of the AAH foci and also the tubular $A C$ (small arrow), which is attached to the CCAM cyst wall. (B) One of the AAH foci enlarged (large arrow in A). (Hematoxylin and eosin; original magnification: $A, \times 25, B, \times 100$.)

FIGURE 4. Mucinous BAC arising outside a CCAM cyst (patient 4). (Hematyoxylin and eosin; original magnification $\times 200$.)

FIGURE 5. Mixed mucinous and nonmucinous BAC adjacent to a CCAM cyst (patient 5). (Hematoxylin and eosin; original magnification $\times 100$.) 

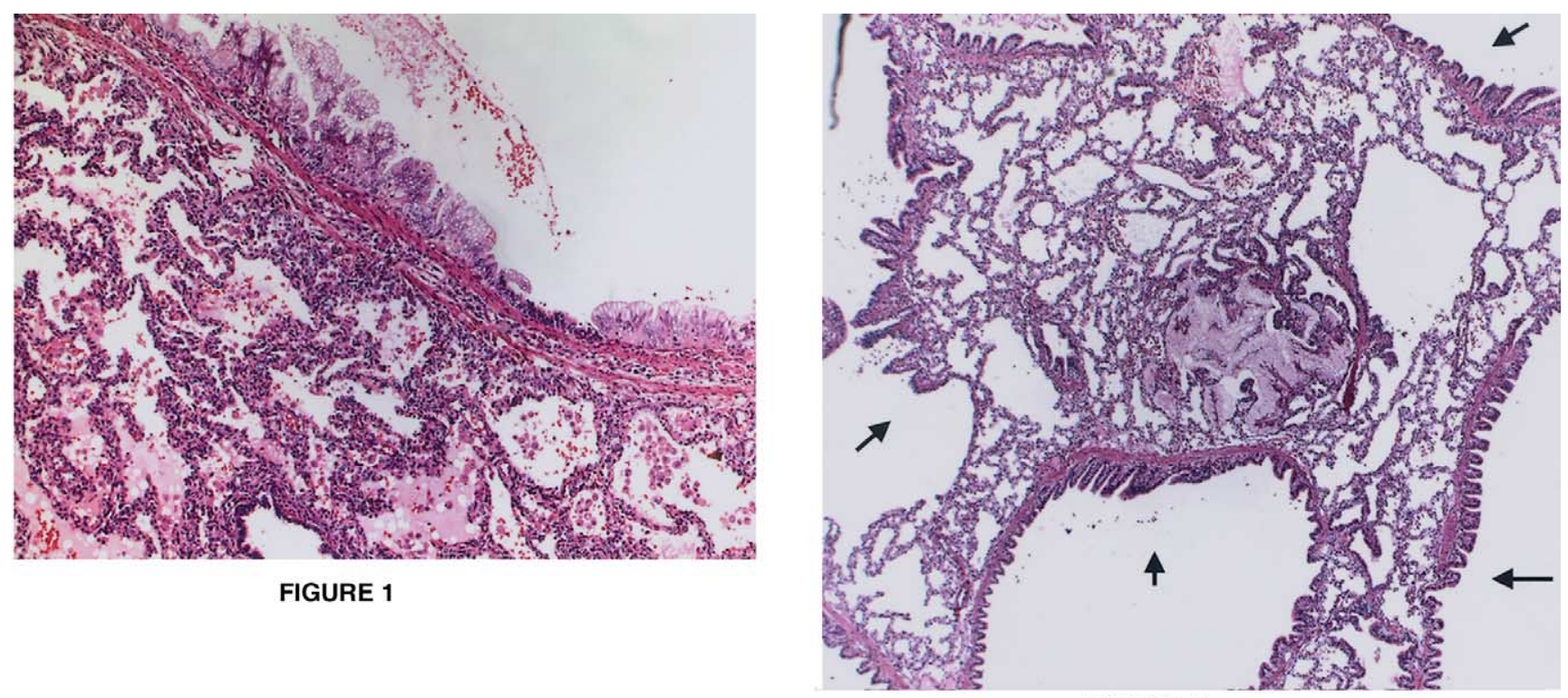

FIGURE 2

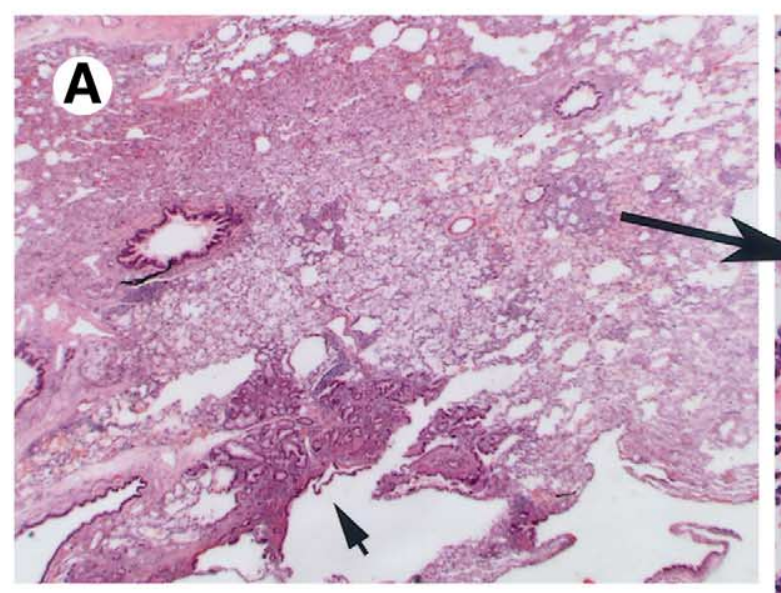

FIGURE 3

B trine 4 .

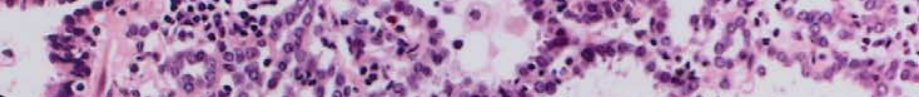
1.

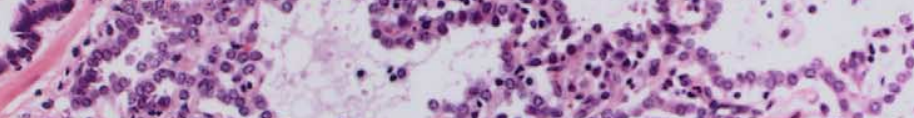

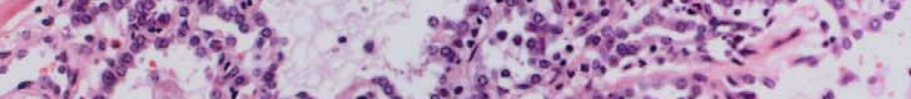

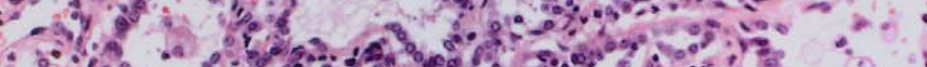

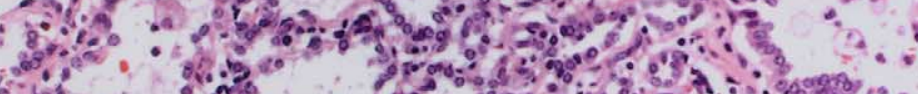
*3. 6.

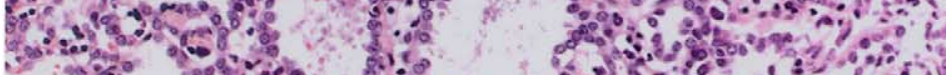
\% 3.

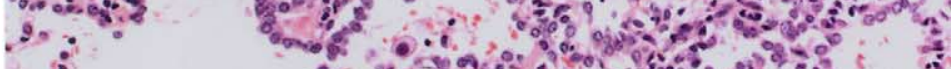

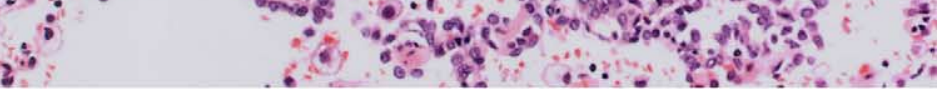

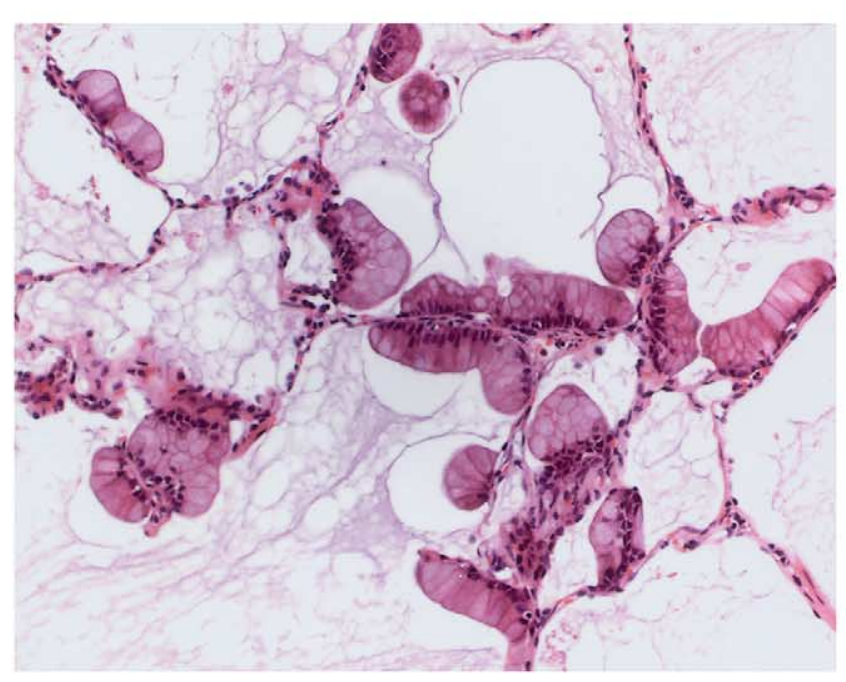

FIGURE 4

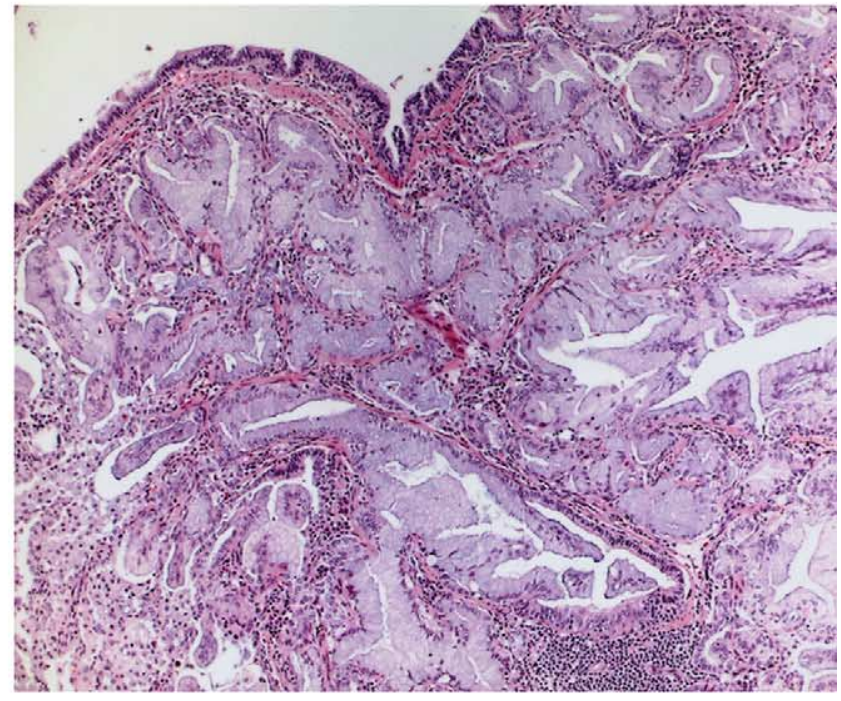

FIGURE 5 


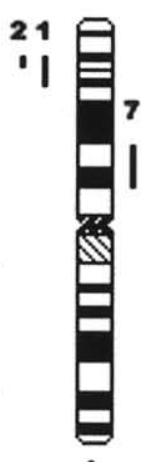

1

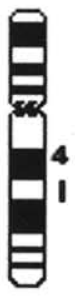

7

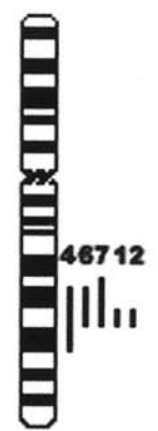

2

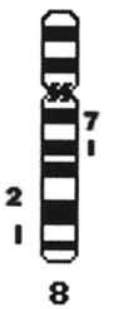

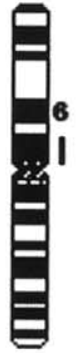

3

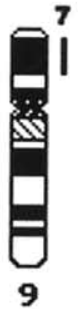

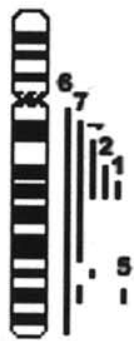

4

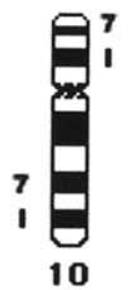

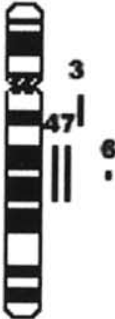

5

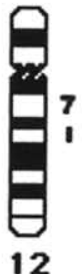

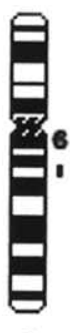

6

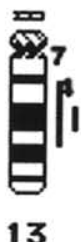

FIGURE 6. Summary of chromosomal aberrations in $A G C H, A A H$ and $A C$, with gains on the right side and losses on the left side. 1, $\mathrm{AGCH} ; 2$, AGCH; 3, focus 1 of $\mathrm{AAH} ; 4$, pooled foci 2 to 5 of $\mathrm{AAH} ; 5$, nonmucinous BAC; 6, mucinous BAC; 7, tubular AC.

CCAM type 1 were associated with AGCH (Figs 1 and 2). These lesions were found within the cysts and also extending into the surrounding lung. One case was combined with a multifocal AAH (Fig 3), 1 case was combined with a tubular AC (Fig 4), and 2 cases were combined with a mucinous and nonmucinous BAC (Fig 5).

Chromosomal aberrations were not detected in the epithelium or in the surrounding normal lung tissue. Both cases of AGCH demonstrated aberrations detected by CGH (Fig 6). Four of the 5 AAH foci (all from patient 3 ) had to be pooled because of their small size. Predominantly gains, but sometimes losses, were found in AGCH, AAH, and AC (Fig 6).

\section{Immunohistochemistry}

IL-13 exhibited a positive nuclear reaction in the atypical goblet cells and a positive cytoplasmic and nuclear reaction in the adjacent epithelium as well as in infiltrating lymphocytes and plasma cells (Fig 7A and B). In addition, macrophages and pneumocytes demonstrated a positive nuclear and cytoplasmic reaction.

IL-4R $\alpha$ and Muc2 were exclusively positive in the nuclei of atypical goblet cells (Fig 8A), whereas in controls a positive cytoplasmic and nuclear reaction for IL-4R $\alpha$ and Muc2 was seen in pneumocytes and some leukocytes (Fig 8B). Bronchiolar (CCAM) epithelium was negative for Muc2, but demonstrated cytoplasmic and nuclear positivity using Il $4 \mathrm{R} \alpha$ antibodies.

\section{DISCUSSION}

No chromosomal aberrations were detected in either epithelium lining cysts or surrounding normal tissues. One reason for this might be that CCAM results from regional deregulation of involved genes at a specific time point during organogenesis and differentiation, not by chromosomal imbalances. This process likely produces the different types of CCAM; for example, type 1 arises from cessation of development at the saccular stage. Point mutations and other alterations that cannot be detected by CGH may also account for this abnormal differentiation.

The AGCHs demonstrated loss in chromosome 1 and gains in chromosomes 2 and 4 . In addition, 1 case of goblet cell proliferation showed gain in chromosome 5 and loss in chromosome 8 .

However, the most frequent genomic imbalances were gains in chromosomes 2 and 4 . These are apparently the initial chromosomal imbalances occurring during carcinogenesis. Therefore, despite the small number of cases, our study indicates that AGCH is a likely precursor to ACs arising from CCAMs, because the same aberrations were also detected in the sampled ACs.

It is also interesting that chromosomal gains were more common than losses. In a study on ACs from nonsmokers, ${ }^{13}$ gains also predominated among the chromosomal aberrations. Another study based on unselected AC cases, supposedly predominantly smokers, found that gains and losses were almost equally present. ${ }^{14}$ Thus dif- 

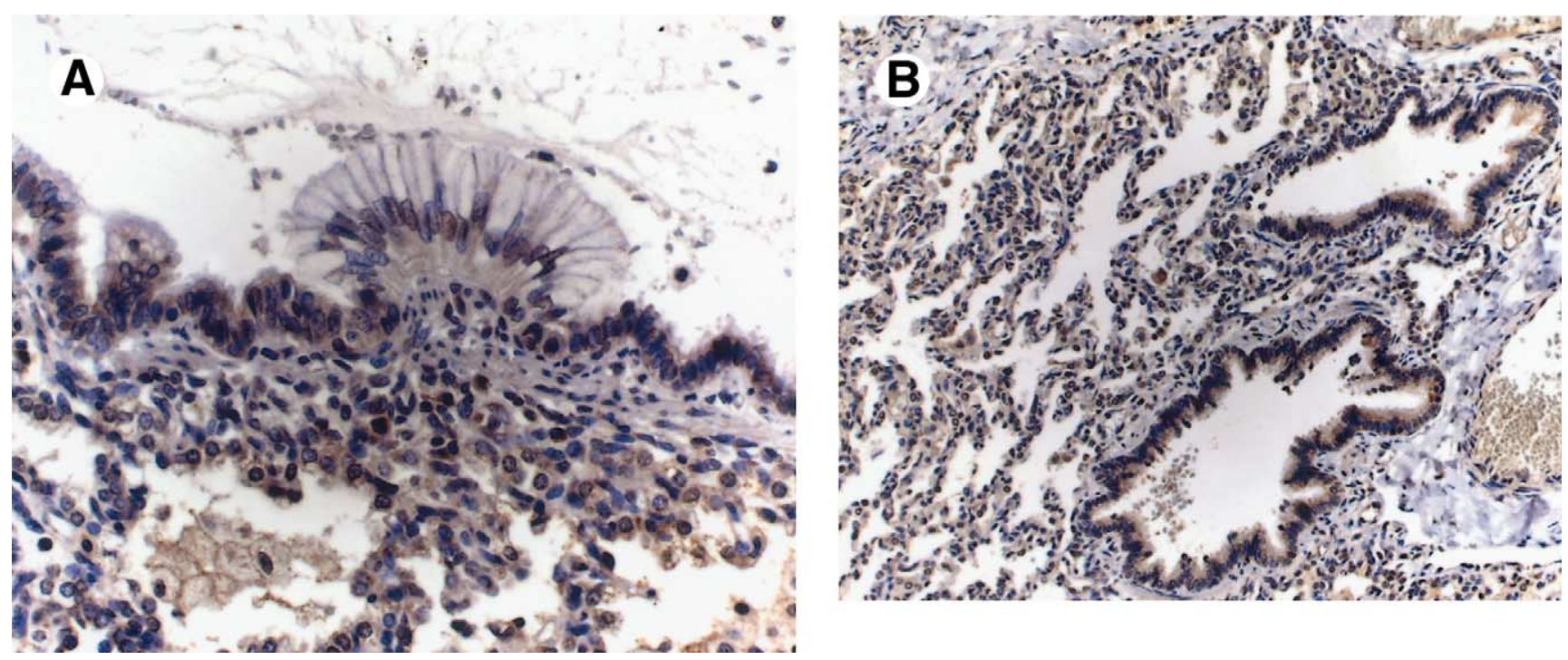

FIGURE 7. Immunohistochemistry for IL-13. (A) Exclusive nuclear staining in $A G C H$, with staining of nuclei and cytoplasm in adjacent normal epithelium as well as inflammatory cells. (B) Normal lung parenchyma outside CCAM, with an intense cytoplasmic as well as nuclear staining for IL-13 antibodies. (Original magnification: $A, \times 400 ; B, \times 100$.)

ferent molecular pathways appear to be involved in the development of AC in smokers, nonsmokers, and childhood carcinomas arising in CCAM.

Another interesting finding was the exclusively nuclear staining for IL-4R $\alpha$ and Muc2 stimulated by IL-13. In contrast to internal controls outside CCAM, where IL-4R $\alpha$ and Muc2 could be demonstrated in the cytoplasm, cell membrane, and nucleus, the staining in AGCH was exclusively nuclear. Therefore, nuclear translocation of the IL-4R $\alpha$ and up-regulation of Muc2 not only may be responsible for goblet differentiation, but also may be involved in neoplastic transformation, similar to the role that has been described for epidermal growth factor receptor. ${ }^{15}$

In conclusion, whereas the respiratory-type surface epithelium in CCAMs does not demonstrate chromosomal aberrations detectable by $\mathrm{CGH}$, these aberrations are present in putative precursor lesions (AGCH and $\mathrm{AAH}$ ). Common genetic aberrations of putative precursor lesions and fully developed ACs are gains on chro-
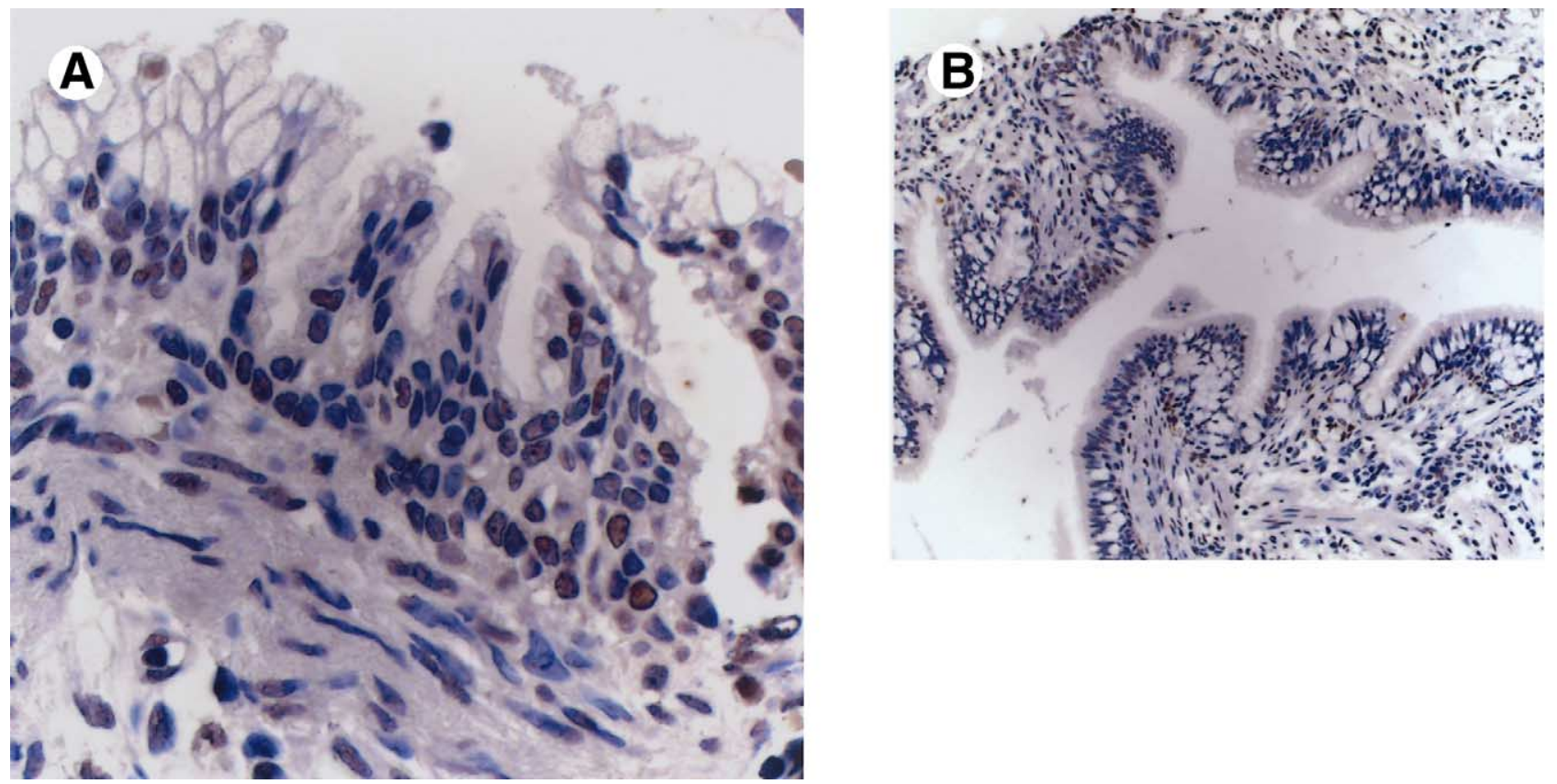

FIGURE 8. Nuclear staining for Muc2 protein in $A G C H(A)$, and focal cytoplasmic and nuclear staining in normal bronchial epithelium and stroma cells (B). (Original magnification: $A, \times 400 ; B, \times 200$.) 
mosomes 2 and 4 . This again stresses the importance of complete resection of the cysts.

Acknowledgment. We gratefully acknowledge support from the Styrian Cancer Society Foundation, the medical faculty of Karl Franzens University of Graz, the Hans und Blanca Moser Foundation, and the Austrian Research Fund (project P14759, to H.H.P.).

\section{REFERENCES}

1. van Dijk C, Wagenvoort CA: The various types of congenital adenomatoid malformation of the lung. J Pathol 110:131-134, 1973

2. Stocker JT, Madewell JE, Drake RM: Congenital cystic adenomatoid malformation of the lung. Classification and morphologic spectrum. Hum Pathol 8:155-171, 1977

3. Stocker JT: Congenital and Developmental Diseases: Pulmonary Pathology. Berlin, Springer-Verlag, 1994

4. Lujan M, Bosque M, Mirapeix RM, et al: Late-onset congenital cystic adenomatoid malformation of the lung. Embryology, clinical symptomatology, diagnostic procedures, therapeutic approach and clinical follow-up. Respiration 69:148-154, 2002

5. Winters WD, Effmann EL: Congenital masses of the lung: Prenatal and postnatal imaging evaluation. J Thorac Imaging 16:196206, 2001

6. MacSweeney F, Papagiannopoulos K, Goldstraw P, et al: An assessment of the expanded classification of congenital cystic adenomatoid malformations and their relationship to malignant transformation. Am J Surg Pathol 27:1139-1146, 2003

7. Travis WD: Congenital Anomalies and Pediatric Disorders Washington, DC, Armed Forces Institute of Pathology, 2002

8. Prichard MG, Brown PJ, Sterrett GF: Bronchioloalveolar carcinoma arising in longstanding lung cysts. Thorax 39:545-549, 1984

9. Kondo M, Tamaoki J, Takeyama K, et al: Interleukin-13 induces goblet cell differentiation in primary cell culture from guinea pig tracheal epithelium. Am J Respir Cell Mol Biol 27:536-541, 2002

10. Rose MC, Piazza FM, Chen YA, et al: Model systems for investigating mucin gene expression in airway diseases. J Aerosol Med 13:245-261, 2000

11. Kallioniemi A, Kallioniemi OP, Sudar D, et al: Comparative genomic hybridization for molecular cytogenetic analysis of solid tumors. Science 258:818-821, 1992

12. Ullmann R, Bongiovanni M, Halbwedl I, et al: Is high-grade adenomatous hyperplasia an early bronchioloalveolar adenocarcinoma? J Pathol 201:371-376, 2003

13. Wong MP, Fung LF, Wang E, et al: Chromosomal aberrations of primary lung adenocarcinomas in nonsmokers. Cancer 97 1263-1270, 2003

14. Petersen I, Bujard M, Petersen S, et al: Patterns of chromosomal imbalances in adenocarcinoma and squamous cell carcinoma of the lung. Cancer Res 57:2331-2335, 1997

15. Lin SY, Makino K, Xia W, et al: Nuclear localization of EGF receptor and its potential new role as a transcription factor. Nat Cell Biol 3:802-808, 2001 